\title{
La vista de información en el modelado del proceso de planificación colaborativa de una RdS/D: diseño de un modelo informacional genérico integrado Information view model within a collaborative planning process modeling for supply and distribution chains: design of an integrated and generic information model
}

\author{
Andrés Boza, Rosa Navarro y Eduardo Vicens \\ Centro de Investigación en Gestión e Ingeniería de la Producción (CIGIP). Universidad Politécnica de Valencia. Camino de Vera, \\ s/n. Ed. 8 G - Esc. 4 - Nivel I. Ciudad Politécnica de la Innovación. 46022 Valencia \\ aboza@cigip.upv.es ronava@doctor.upv.es evicens@cigip.upv.es
}

Fecha de recepción: 06-10-08

Fecha de aceptación: 02-12-08

Resumen. Esta comunicación tiene por objeto destacar el papel de la Vista Informacional como elemento integrador en el modelado del proceso de Planificación de la Producción de una Red de Suministro y Distribución. Para ello, tomando como punto de partida la definición de Vista Informacional (Boza et al., 2007) adoptada en el contexto del Marco Conceptual del proyecto CICYT RdS-2V.RDSINC, y basados en la Metodología definida por Lario et al. (2007), se presentan, a través de diagramas de clases UML, los modelos informacionales individuales (parciales) correspondientes a la Vista de Recursos, la Vista Organizacional y la Vista Decisional del proceso abordado. Se destaca la utilidad del desarrollo de dichos modelos, los cuales permiten llegar a un modelo informacional integrado resultante de las interconexiones entre elementos o clases de los modelos informacionales individuales. Finalmente se describen particularidades y aportes del proceso de desarrollo del modelo informacional integrado, entre los que se destacan los beneficios de reunir en un único diagrama estructurado la información relevante del proceso Planificación Colaborativa de una RdS/D.

Palabras clave: modelado empresarial, vista de información, planificación colaborativa, UML.

Abstract. The purpose of this paper is to draw attention to the role of the Information View as a link in the Collaborative Planning Process Modeling for a Supply and Distribution Chain. Starting from the Information View definition (Boza et al., 2007) in the concept framework of CICYT RdS-2V.RDSINC project (Lario et al., 2007), we developed independent UML information models, corresponding to the resource, organization, and decision views of the above mentioned process. This allowed us to work on an integrated information model, in which the connections between the different models were identified and became visible through the associations between UML classes of different views in a UML integrated information model. Finally, we review contributions of the integrated information model. One of the most important contributions is that it gathers all the relevant information of the Collaborative Planning Process for Supply and Distribution Chains in a unique structured diagram.

Key words: enterprise modeling, information view, collaborative planning, UML.

\section{Introducción}

La Metodología para la determinación del Entorno Decisional de un Centro de Decisión genérico en un contexto jerárquico de Planificación Colaborativa de una Red de Suministro/Distribución (RdS/D), definida por Lario et al. (2007), tiene como objeto la construcción de Modelos Analíticos que ayuden a la Toma de Decisiones en el Proceso de Planificación
Colaborativa de una RdS/D, todo ello en un contexto jerárquico, por tratarse de procesos de Planificación Táctico-Operativos. Entre los elementos necesarios para el modelado de dicho proceso, esta metodología cuenta con cuatro visiones: Recursos, Organizacional, Decisional e Informacional.

En este contexto, cada vista debe ser capaz, desde su correspondiente perspectiva, de abstraer los da- 
tos de la realidad necesarios para llevar a cabo el Proceso de Planificación Colaborativa de una RdS/D. En particular, la Vista de Información debe proporcionar las estructuras de datos que sirvan de soporte a dicho proceso. La identificación de estas estructuras y su nivel de agregación quedará condicionada a la descripción, del proceso en cuestión, que aporten el resto de vistas.

El objetivo de esta comunicación es destacar el papel de la Vista Informacional como elemento integrador en el modelado del proceso de Planificación de la producción de una Red de Suministro y Distribución*.

\section{La vista de Información}

Las arquitecturas de modelado de empresa proporcionan un conjunto de vistas de modelado para focalizar y trabajar en una parte específica del modelo de empresa integrado, en contraposición a manejar un único y complejo modelo completo (Toh, 1999). Cada arquitectura de modelado propone sus propias vistas de modelado, por ejemplo:

- CIMOSA: Vistas de Organización, Recursos, Información y Función.

- GRAI-GIM: Vistas del Sistema Físico, Decisional, de Información y Funcional.

- PERA: Arquitectura de Organización y RR.HH., del Sistema de Información y del Equipo de Producción.

- GERAM:Vistas de Organización, Recursos, Información y Función.

- ARIS: Vistas de Función, Datos, Organización y Control.

- Zachman: Datos, Función, Localización, Personas, Tiempo, Motivación.

- TOGAF: Visión Arquitectónica, Arquitectura de Negocio, del Sistema de Información y Tecnológica.

Las arquitecturas proceden de distintas áreas de conocimiento. Por ello, algunas arquitecturas están más orientadas al modelado de procesos empresariales y su posible re-ingeniería (CIMOSA, GRAI, ISO/DIS 19439), y otras más orientadas a la implementación de tecnologías de la información en la empresa (ARIS) (Boza et al., 2007).

Una comparativa entre las vistas propuestas por diferentes arquitecturas se puede encontrar en Cuenca et al. (2005) y en Tang et al. (2004).

Un punto de coincidencia en las arquitecturas de modelado empresarial es la vista de información o datos. Aunque como se puede ver, la vista de información aparece como una componente común en las arquitecturas, no todas la abordan desde la misma perspectiva, adoptando diferente significado para cada una de ellas.

Por ejemplo, en CIMOSA, la vista de información representa objetos de empresa y elementos de información. Para la arquitectura GRAl, el sistema de información une el sistema de decisión con el sistema físico y el entorno de la empresa. Para ello transforma y almacena información. En ARIS, la vista de datos se utiliza para definir modelos de datos semánticos (mediante diagramas Entidad-Relación), para posteriormente pasarlos a un esquema Relacional antes de implementarlos en una base de datos. Y el estándar de integración empresarial ISO/DIS 19439 define la vista de información como aquella que describe los objetos de la empresa relacionados con la información -information related enterprise objects- (materiales y de información), y cómo son utilizados y producidos en el curso de las operaciones de la empresa (Vernadat, 1995).

Así, para unas arquitecturas la vista de información se trata de forma estática, utilizándose únicamente para la representación de las estructuras de información y su almacenamiento, este enfoque aparece en CIMOSA y ARIS donde la vista de información se limita a la identificación y representación de los objetos de información. Para otras arquitecturas la vista de información tiene un sentido dinámico donde además de la identificación y representación de los objetos de información, también se incorpora el procesamiento y transformación de la información, es el caso de las arquitecturas GRAI e ISO/DIS 19439 (Boza et al., 2007).

Las técnicas para representar las vistas son variadas. En Gaiglis (200I), Aguilar-Saven (2004) y Shen et al. (2004) se puede encontrar técnicas de modelado

\footnotetext{
* Este trabajo se deriva de la participación de sus autores en un proyecto de investigación Feder-Cicyt titulado «De la planificación a la ejecución en la Cadena (Red) de Suministro. Dos visiones diferentes y sus herramientas». RdS-2V.RDSINC.
} 
para la representación de las diferentes vistas. Una de las técnicas que se repite para la representación de la vista de información en los trabajos anteriormente citados es UML (Unified Modeling Language).

La técnica de modelado UML es la que los autores de este trabajo hemos elegido para el modelado de la vista de información del Proceso de Planificación Colaborativa de una RdS/D. Uno de los diagramas más representativos proporcionados por UML es el diagrama de clases. Este diagrama ha permitido modelar la vista de información como un conjunto de clases y sus relaciones que representan tanto los aspectos físicos como abstractos del Proceso de Planificación Colaborativa de una RdS/D.

\section{La vista de información en el modelado del Proceso de Planificación Colaborativa de una RdS/D}

Siguiendo la metodología definida por Lario et al. (2007), que tiene como objeto la construcción de Modelos Analíticos que ayuden a la Toma de Decisiones en el Proceso de Planificación Colaborativa de una RdS/D, se establecen como elementos necesarios para el modelado de dicho proceso cuatro visiones: Recursos, Organizacional, Decisional e Informacional.

Los modelos realizados para las vistas de recursos, organizacional y decisional han sido utilizados en la construcción de la vista informacional. Estos modelos se han desarrollado en el contexto del proyecto CICYT RdS-2V.RDSINC y aparecen parcialmente en Perez et al. (2007), Alemany et al. (2007), Alarcon et al. (2007) y Boza et al. (2007) en forma descriptiva o representados mediante herramientas de modelado como IGRAF o IDEF.

En una primera etapa, se han desarrollado los modelos informacionales en UML para cada una de las vistas (recursos, decisional y organizacional) a partir de los diferentes modelos desarrollados previamente para cada una de ellas. De esta forma se han obtenido tres modelos informacionales desarrollados en UML, de las vistas de recursos, decisional y organizacional. El nivel de detalle del modelo informacional de cada vista ha venido condicionado por el nivel de detalle proporcionado por los modelos realizados previamente para cada una de estas.

Estos modelos informacionales son complejos y su descripción va más allá del propósito de esta comunicación. Aunque, para una mejor comprensión se presenta a continuación algunos de los elementos más destacados en cada modelo informacional.

\section{I. Modelo informacional de la vista de recursos}

La vista de recursos recoge todos los aspectos físicos a tener en cuenta a la hora de la toma de decisiones del proceso de planificación colaborativa en contextos de RdS/D.

Una cadena de suministro incluye una serie de etapas. Estas etapas incluyen por ejemplo, clientes, distribuidores, fabricantes, suministradores de materia prima, y cada uno de ellos aparece en una determinada posición siguiendo un orden concreto para cada cadena de suministro. El siguiente diagrama UML muestra esta estructura:

Figura I

Definición informacional de la vista de recursos

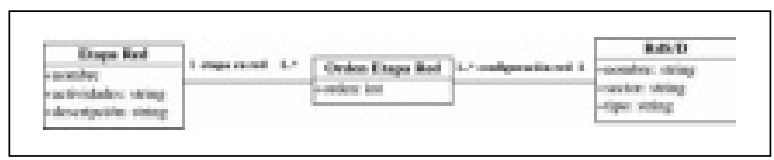

Otras clases que aparecen en este modelo son: Nodo (definido como instalación física en general donde pueden aparecer nodos de producción-operaciones, nodos de almacenamiento, etc.), Centros de Trabajo, Sección y Líneas de Producción.

Figura 2

Nodo y sus posibles configuraciones

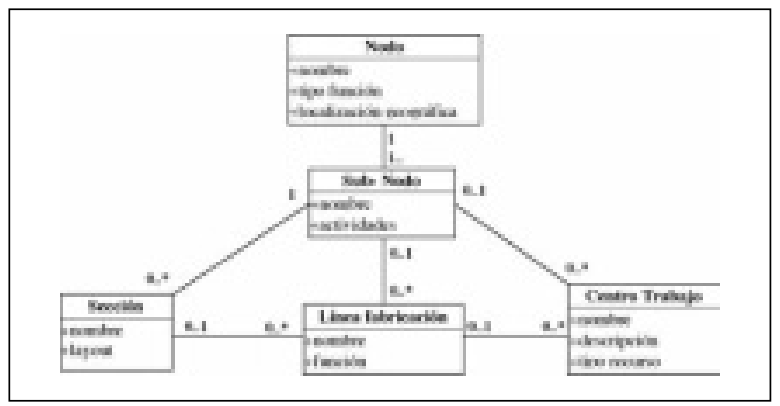

\subsection{Modelo informacional de la vista de organización}

La visión organizacional hace referencia a cómo se organizan los recursos dentro de la red de suministro/distribución y qué tipo de relaciones existen entre ellos.

En un nivel micro organizacional el nodo se divide en centros organizacionales que colaborarán conjunta- 
Figura 3

Relaciones entre centros organizacionales y centros inter-organizacionales en un determinado nivel de gestión

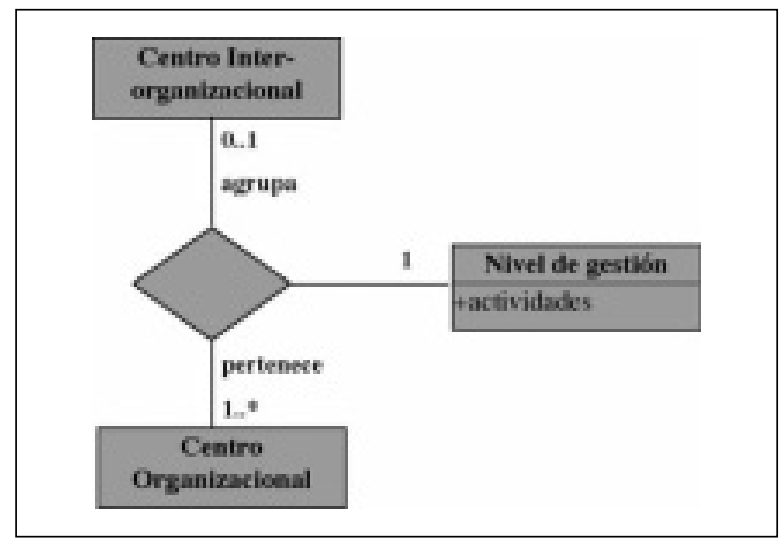

mente con otros centros de otros nodos para llevar a cabo el proceso de planificación colaborativa.

En un nivel macro-organizacional se definen los centros inter-organizacionales los cuales pueden actuar y tomar las decisiones para un grupo de centros organizacionales, colaborar con otros centros inter-organizacionales o depender de otros centros inter-organizacionales.

Estos centros inter-organizacionales y centros organizacionales siempre operarán en un nivel organizacional, ya sea operativo, táctico o estratégico.

\subsection{Modelo informacional de la vista de decisión}

Las decisiones y cómo son tomadas en el Proceso de Planificación Colaborativa de una RdS/D se incluyen en la vista decisional.

En una organización, es posible encontrar centros de decisión, de forma que un centro organizacional o inter-organizacional puede considerarse como un centro de decisión dentro de la red/cadena de suministro.

Se han definido dos tipos de centros de decisión: superior y base. Los centros de decisión superiores son los que inician el proceso de toma de decisiones, mientras que los base no inician ningún proceso de toma de decisiones.

Los centros de decisión interactúan con otros centros para llegar a un mutuo acuerdo en la toma de decisiones. El tipo de interacción será jerárquica cuando no todos los centros de decisión tienen el mis-
Figura 4

Interacción entre centros de decisión

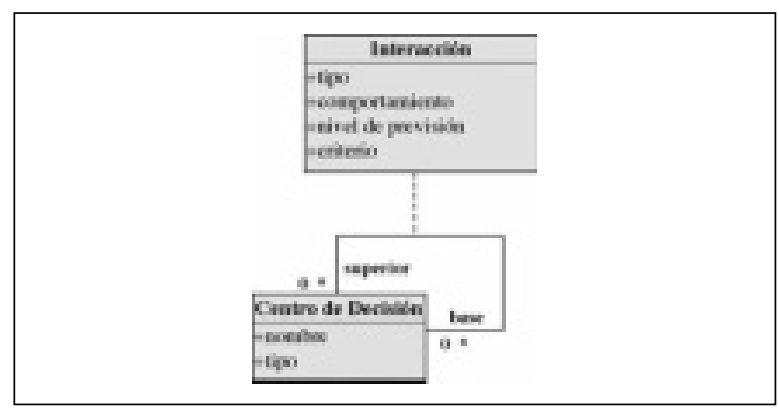

mo peso a la hora de tomar la decisión final, existiendo, por tanto, cierto tipo de jerarquía, y será no jerárquica cuando todos los centros de decisión tienen el mismo peso a la hora de tomar una decisión final, y, por tanto, la decisión final se toma por consenso.

\subsection{Utilidad del desarrollo de los modelos informacionales de cada vista}

El desarrollo de los modelos informacionales, a partir de los modelos de cada una de las vistas, ha permitido realizar una revisión de conceptos y estructura de estos últimos. De forma que las reuniones llevadas a cabo entre los diseñadores de los modelos pertenecientes a la vista de recursos, decisional y organizacional, junto con los diseñadores del modelo informacional, han servido como feedback de los modelos iniciales y el enriquecimiento del mismo con los aportes desde la perspectiva informacional.

Los diagramas de clases se documentan con la descripción de las clases, sus métodos y atributos. Por este motivo, el diseño del modelo informacional ha obligado a profundizar en el resto de modelos en aquellos aspectos donde se requería de un mayor nivel de detalle en su descripción.

\subsection{Modelo de información integrado}

El desarrollo de los modelos informacionales pertenecientes a cada una de las vistas, ha permitido trabajar en un modelo informacional integrado.

En este sentido, las conexiones entre los diferentes modelos se fueron identificando, es decir, las conexiones entre las diferentes vistas. Dichas conexiones se materializaron a través de asociaciones (relaciones) entre clases UML entre los modelos informacionales de la vista de recursos, decisión y organización. 
Figura 5

Modelo informacional integrado

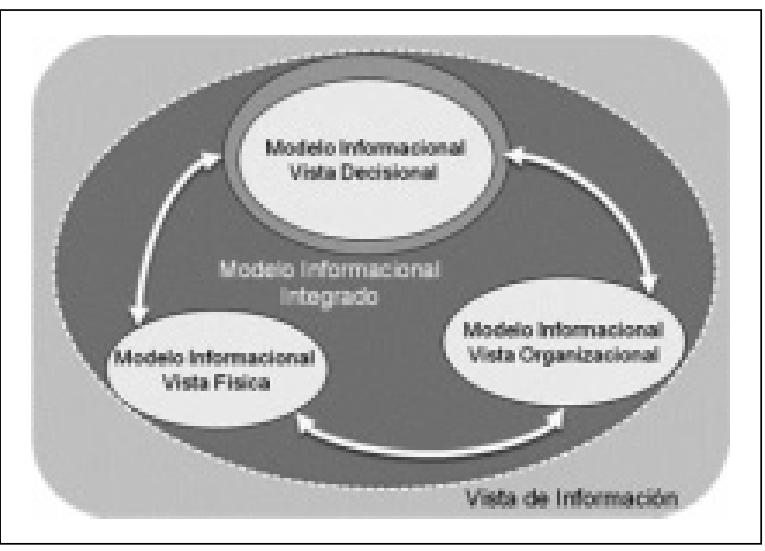

Como resultado, se realizó un modelo de información integrado en el cual es posible apreciar la contribución de las diferentes vistas al proceso de Planificación Colaborativa de una RdS/D (Figura 5).

El propósito de mostrar el modelo informacional integrado no es detenernos en detalles particulares del mismo, sino enfatizar las interconexiones entre las vistas, las cuales han sido incluidas como asociaciones entre clases UML (Figura 6).

\subsection{Desarrollo del modelo informacional integrado}

Tanto los modelos informacionales de cada vista como el modelo integrado se han ido rediseñando paralelamente al rediseño de cada vista. En otras palabras, a medida que se han madurado y completado los conceptos de las diferentes vistas, estos han sido incluidos en el correspondiente modelo informacional.

El desarrollo del modelo informacional integrado ha permitido llegar a un vocabulario consensuado entre los distintos diseñadores ya que, en ocasiones, se identificaba a los mismos elementos del proceso con distinta nomenclatura. Para ello se ha utilizado el documento que acompaña al diagrama de clases, con la relación de clases y su descripción, como herramienta para la identificación y descripción única de elementos que podían aparecer en varios modelos.

Esta labor ha proporcionado un importante feedback entre modelos, al representarse sobre un único modelo los aspectos informacionales del proceso Planificación Colaborativa de una RdS/D procedentes de las distintas vistas.

Modelo informacional integrado en UML

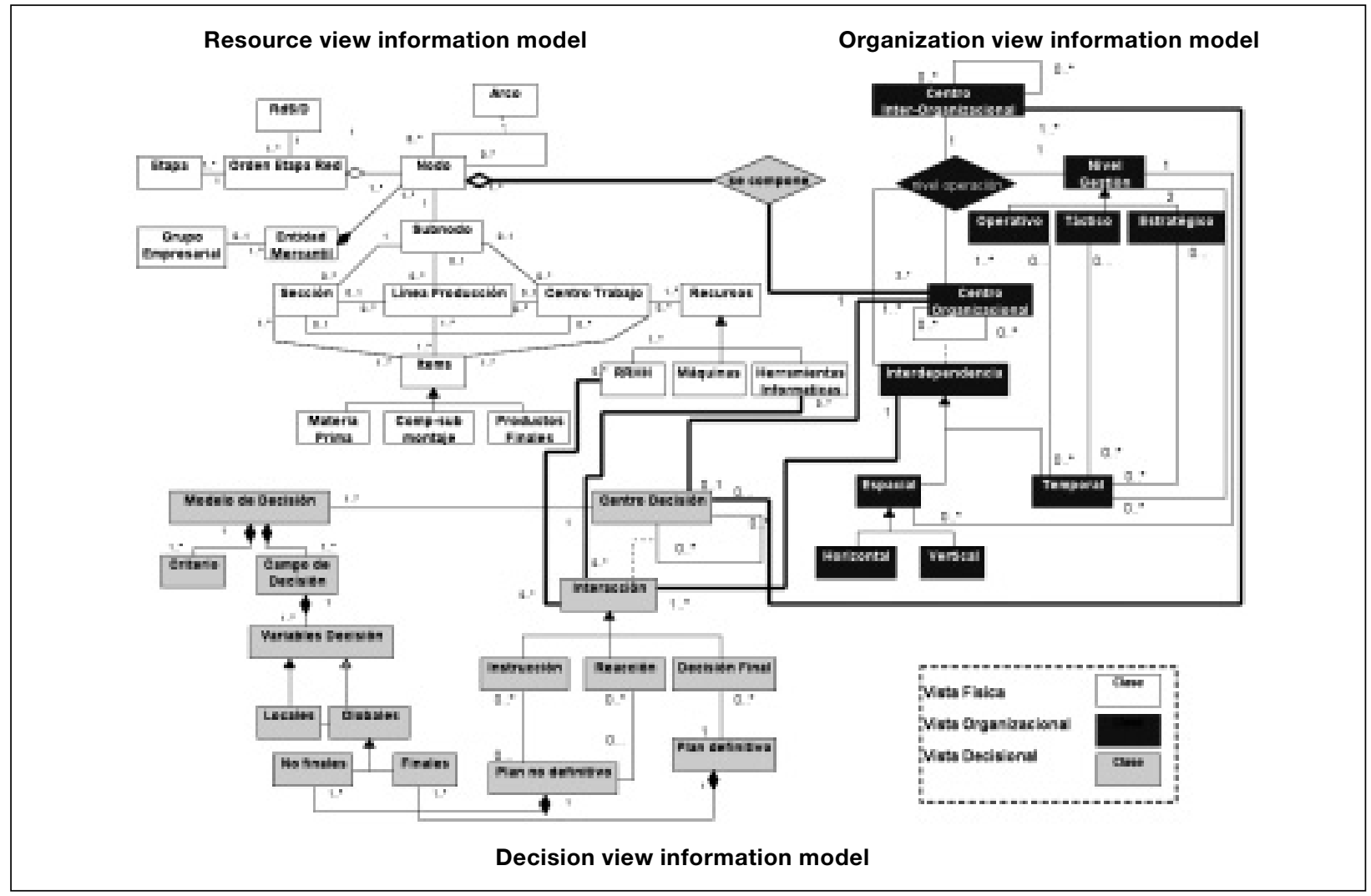




\section{Conclusiones y trabajo futuro}

Como conclusión se destaca la utilidad de la Vista Informacional como elemento conector entre vistas. El modelo informacional permite recoger en un único diagrama estructurado la información relevante del proceso Planificación Colaborativa de una RdS/D.

El modelo informacional ha sido desarrollado en un nivel genérico, de forma que pueda ser utilizado en la definición del modelo informacional del Proceso de Planificación Colaborativa de una RdS/D particular.

El empleo de un lenguaje de modelado como UML proporciona un punto de partida para definir las estructuras de datos necesarias para el desarrollo de una herramienta de ayuda a la toma de decisiones para el proceso de planificación colaborativa. En este sentido, debe proporcionar todos los datos necesarios para la resolución del modelo analítico de decisión.

El proceso de definición del modelo informacional, que integra al resto de modelos, ha proporcionado un importante feedback entre los mismos, y ha puesto de manifiesto la necesidad de unificar criterios en cuanto a definición de conceptos y terminología utilizada en las diferentes vistas entre los diseñadores de los distintos modelos.

En este proyecto se ha considerado la Vista Informacional desde un punto de vista estático, en el sentido que considera sólo las estructuras de datos (clases UML). Un objetivo a futuro es ampliar el modelado de la Vista Informacional, para el proceso bajo estudio, incluyendo la componente dinámica. Esto es, modelar la forma en que dichos datos son generados y transformados.

\section{Referencias}

AGUILAR-SAVEN, R.S. (2004). «Business process modelling: Review and framework». International Journal of Production Economics, 90 (2), pp. I29- I 49.

ALARCÓN, F.; LARIO, F.C.; BOZA, A., Y PÉREZ, D. (2007). Propuesta de marco conceptual para el modelado del proceso de planificación colaborativa de operaciones en contextos de Redes de Suministro/Distribución (RdS/D). XI Congreso de Ingeniería de Organización, Madrid.

ALEMANY, M.M.; PÉREZ, D.; ALARCÓN, F., y BOZA, A. (2007). Planificación colaborativa en cadenas de suministro mediante programación matemática en entornos distribuidos. XI Congreso de Ingeniería de Organización, Madrid.
BOZA, A.; ALARCÓN, F.; VICENS, E., y ALEMANY, M. (2007). Propuesta de marco conceptual para el modelado del proceso de planificación colaborativa de una Red de Suministro/Distribución (RdS/D). Visión Informacional. XI Congreso de Ingeniería de Organización, Madrid.

CHEN, D., y VERNADAT, F. (2004). «Standars on enterprise integration and engineering. State of the art.». International Journal of Computer Integrated Manufacturing, 17 (3), pp. 235-253.

CHOPRA, S., y MEINDL, P. (200I). Supply chain management: strategy, planning, and operation. NY: Prentice Hall.

CUENCA,LL.; ORTIZ, A., y BOZA, A. (2005). Arquitectura de empresa. Visión general. IX Congreso de Ingeniería de Organización, Madrid,

DE KOK, A.G., y FRANSOO, J.C. (2003). «Planning supply chain operations: definition and comparison of planning conceptsy. In: Handbooks in Operations Research and Management Science Series. Amsterdam: North Holland.

ERENGUC, S.S.; SIMPSON, N.C., YVAKHARIA, A. J. (1999). «Integrated production/distribution planning in supply chains: an invited review». European Journal of Operational Research, II 5 (2), pp. 219-236.

FLEISCHMANN, B., y MEYR, H. (2002). Planning hierarchy, modeling and advanced planning. Amsterdam: North-Holland.

GIACHETTI, R.E. (2004). «A framework to review the information integration of the enterprise». International Journal of Production Research, 42 (6), pp. I I 47- I 166.

GIAGLIS, G.M. (200I). «A taxonomy of business process modeling and information systems modeling techniques». International Journal of Flexible Manufacturing Systems, 13 (2), pp. 209-228.

LARIO, F.; PÉREZ, D.;ALEMANY, M., y ALARCÓN, F. (2007). Propuesta de marco conceptual para el modelado del proceso de planificación colaborativa de operaciones en contextos de Redes de Suministro/Distribución (RdS/D). XI Congreso de Ingeniería de Organización, Madrid.

NEAGA, E.I., y HARDING, J.A., (2005). «An enterprise modeling and integration framework based on knowledge discovery and data mining». International Journal of Production Research, 43 (6), 1089-1 108.

PÉREZ, D.; ALEMANY, M.M.; VICENS, E., y LARIO, F.C. (2007). Propuesta de marco conceptual para el modelado de la visión decisional del proceso de planificación colaborativa de una Red de Suministro/Distribución (RdS/D). XI Congreso de Ingeniería de Organización, Madrid

SCHIEGG, P.; ROESGEN, R.; MITTERMAYER, H., y STICH, V. (2003). "Supply chain management systems A survey of the state-of-the-art». In: Collaborative supply net management. Jagdev, Wortmann, and Pels, eds., IFIP. 
SHEN, H.;WALL, B.; ZAREMBA, M.; CHEN, Y.L.,y BROWNE, J. (2004). «Integration of business modelling methods for enterprise information system analysis and user requirements gathering». Computers in Industry, 54 (3), pp. 307-323.

STADTLER, H., y KILGER, C. (2002). Supply chain management and advanced planning». Springer.

TANG, A.; HAN J.,y CHEN, P. (2004). A comparative analysis of architecture frameworks. Technical Report: SUTITTR2004.0I CeCSES Centre Report: SUT.CeCSESTROOI.
TOH, K.T.K. (1999). «Modelling architectures: a review of their application in structured methods for information systems specication». International Journal of Production Research, 37 (7), pp. | 439- 458.

VERNADAT, F.B. (1996). Enterprise modelling and integration: principles and application. London: Chapman and Hall.

ZORYK-SCHALLA, A.J.; FRANSOO, J.C., y DE KOK, T.G. (2004). «Modeling the planning process in advanced planning systems». Information \& Management, 42 (I), pp. 75-87. 